\title{
NEIGHBORHOODS OF ALGEBRAIC SETS ${ }^{1}$
}

BY

\author{
ALAN H. DURFEE
}

\begin{abstract}
In differential topology, a smooth submanifold in a manifold has a tubular neighborhood, and in piecewise-linear topology, a subcomplex of a simplicial complex has a regular neighborhood. The purpose of this paper is to develop a similar theory for algebraic and semialgebraic sets. The neighborhoods will be defined as level sets of polynomial or semialgebraic functions.
\end{abstract}

Introduction. Let $M$ be an algebraic set in real $n$-space $\mathbf{R}^{n}$, and let $X$ be a compact algebraic subset of $M$ containing its singular locus, if any. An algebraic neighborhood of $X$ in $M$ is defined to be $\alpha^{-1}[0, \delta]$, where $\delta>0$ is sufficiently small and $\alpha: M \rightarrow \mathbf{R}$ is a proper polynomial function for which $\alpha \geq 0$ and $\alpha^{-1}(0)=X$. Such an $\alpha$ will be called a rug function. Occasionally we will need rational or analytic $\alpha$, but this is not a significant generalization. Algebraic neighborhoods always exist. The curve selection lemma is used to prove uniqueness; anyone familiar with [Milnor 2] will recognize the technique. Since uniqueness is a crucial result, and since there are a few troublesome small points, this proof is given in detail. The uniqueness theorem shows that the "link" of a singularity of an algebraic set $M$ is independent of the embedding of $M$ in its ambient space; $I$ have been unable to find a proof of this result in the literature. In addition, an algebraic neighborhood of a nonsingular $X$ in $M$ is shown to be a tubular neighborhood in the sense of differential topology.

This material is in $\S 1$. In $\S 2$ the theory is rapidly developed for real and complex projective space by embedding these spaces in real affine space. As an application, it is shown that when $M$ is an affine algebraic set with projective completion $\bar{M}$, then the complement in $\bar{M}$ of a large ball centered at the origin of affine space is an algebraic neighborhood of the intersection of $\bar{M}$ with the hyperplane at infinity.

In $\S 3$, the theory is generalized to the case where $M$ is a semialgebraic subset of $\mathbf{R}^{n}$ and $X$ is a compact semialgebraic subset of $M$. (For example, $X$ could be a nonisolated singular point of $M$.) A semialgebraic neighborhood of $X$ in $M$ is defined to be $\alpha^{-1}[0, \delta]$, where $\delta>0$ is sufficiently small and $\alpha: M \rightarrow \mathbf{R}$ is a proper semialgebraic function for which $\alpha \geq 0$ and $\alpha^{-1}(0)=X$. Again these neighborhoods exist and are unique; this is shown by mimicking the proof in the algebraic case, using semialgebraic

Received by the editors April 14, 1980 and, in revised form, June 24, 1981.

1980 Mathematics Subject Classification. Primary 14G30, 32B25, 57Q40.

Key words and phrases. Tubular neighborhood, regular neighborhood, rug function, link of singularity, stratification, Milnor fibration theorem, semialgebraic sets.

${ }^{1}$ Research partially supported by National Science Foundation grant MCS 8002839 and MCS7608910. 
stratifications and Thom-Mather isotopy theory. A proof is given of the well-known fact that semialgebraic maps can be stratified (Theorem 3.3). When $X$ and $M$ are simplicial complexes, a PL regular neighborhood of $X$ in $M$ is a semialgebraic neighborhood. In addition, the "polydisk" link of a singularity is shown to be homeomorphic. to the usual link. Also, Milnor's fibration theorem is generalized from polynomials on $\mathbf{C}^{n}$ to polynomials on an arbitrary complex set, as is the theorem on the connectivity of the fiber of this fibration.

$\S 4$ generalizes the theory to semialgebraic neighborhoods of algebraic sets in projective space. The main purpose here is to show that the boundary of a neighborhood of the exceptional set in the resolution of a singularity is homeomorphic to the usual link of a singularity. A similar result is given for singular fibrations.

$\S 5$ contains an example showing that uniqueness does not hold for smooth (nonalgebraic) rug functions.

The idea of a rug function came from [Thom], which develops a theory of smooth neighborhoods of simplicial complexes in $\mathbf{R}^{n}$.

0. Preliminaries. The symbol $\mathbf{A}^{n}$ will denote real affine $n$-space $\mathbf{R}^{n}$ or complex affine $n$-space $\mathbf{C}^{n}$. A subset of $\mathbf{A}^{n}$ is an algebraic set if it is the set of common zeros of some collection $f_{1}, \ldots, f_{k}$ of polynomials. Note that the zero locus of a set of complex polynomials in $\mathbf{C}^{n}$ is also a real algebraic set in $\mathbf{R}^{2 n}$.

Let $X$ be an algebraic set in $\mathbf{R}^{n}$, and suppose that $f_{1}, \ldots, f_{k}$ span the ideal of all polynomials vanishing on $X$. Let $\rho$ be the maximum value of the rank of the $k \times n$ matrix $\left[\partial f_{i}(p) / \partial x_{j}\right]$, over all $p \in X$. A point $p$ is nonsingular or smooth if

$$
\operatorname{rank}\left[\frac{\partial f_{i}}{\partial x_{j}}(p)\right]=\rho .
$$

The set of nonsingular points of $X$ forms a real or complex analytic manifold of dimension $n-\rho$. For further discussion, see [Milnor 2, §2].

The symbol $\mathbf{P}^{n}$ will denote real projective $n$-space $\mathbf{R P}^{n}$ or complex projective $n$-space $\mathbf{C P}^{n}$. Points of projective $n$-space $\mathbf{P}^{n}$ will be written as $(n+1)$-tuples $\left(x_{0}, \ldots, x_{n}\right)$ of real or complex numbers, not all zero, with the equivalence relation $\left(x_{0}, \ldots, x_{n}\right) \sim\left(\lambda x_{0}, \ldots, \lambda x_{n}\right)$ for $\lambda$ a nonzero real or complex number. An algebraic set in $\mathbf{P}^{n}$ is the set of common zeros of some finite collection of homogeneous polynomials in $n+1$ variables. All such sets are compact. Let

$$
H_{i}=\left\{\left(x_{0}, \ldots, x_{n}\right) \in \mathbf{P}^{n}: x_{i}=0\right\} .
$$

Then $\mathbf{P}^{n}-H_{i}$ is isomorphic to $\mathbf{A}^{n}$ by the obvious map. A point $x=\left(x_{0}, \ldots, x_{n}\right)$ of an algebraic set $X$ in $\mathbf{P}^{n}$ with $x_{i} \neq 0$, some $i$, is nonsingular if it is nonsingular in the affine algebraic set $X \cap\left(\mathbf{P}^{n}-H_{i}\right)$.

Let $X \subset \mathbf{P}^{n}$ be a projective algebraic set. A rational function on $X$ is one which may be written as the quotient of two homogeneous polynomials of the same degree. (A (real) rational function on a complex projective algebraic set is one which may be written as the quotient of two homogeneous real polynomials of the same degree.) 
A subset $X$ of $\mathbf{R}^{n}$ is semialgebraic if it can be written as a finite union and intersection of sets of the form $\left\{x \in \mathbf{R}^{n}: f(x)=0\right\}$ and $\left\{x \in \mathbf{R}^{n}: f(x)>0\right\}$, where $f$ is a polynomial function on $\mathbf{R}^{n}$. A semialgebraic set is nonsingular if it is an open subset of the set of nonsingular points of some algebraic set.

Let $X \subset \mathbf{R}^{n}$ and $Y \subset \mathbf{R}^{m}$ be semialgebraic sets. A function $f: X \rightarrow Y$ is semialgebraic if it is continuous and its graph is a semialgebraic subset of $\mathbf{R}^{n} \times \mathbf{R}^{m}$. For example, rational functions are semialgebraic; so is the function $f: \mathbf{R}^{2} \rightarrow \mathbf{R}$ defined by $f(x, y)=\max (x, y)$. Finite simplicial complexes in $\mathbf{R}^{n}$ are semialgebraic sets, and simplicial maps are semialgebraic functions. The Tarski-Seidenberg theorem states that the image of a semialgebraic set under a semialgebraic map is again semialgebraic. Hence the composition of two semialgebraic functions is again semialgebraic.

Semialgebraic sets can be triangulated (see, for instance, [Hironaka, p. 170]): Let $X_{1}, \ldots, X_{r}$ be semialgebraic sets in $\mathbf{R}^{n}$. Then there is a locally finite simplicial decomposition of $\mathbf{R}^{n}=\bigcup \Delta_{i}$ into open simplexes and a semialgebraic automorphism $\sigma$ of $\mathbf{R}^{n}$ such that each $X_{k}$ is a finite union of some of the $\sigma\left(\Delta_{i}\right)$.

Let $X \subset \mathbf{P}^{n}$ be an algebraic set. A function $f: X \rightarrow \mathbf{R}$ is semialgebraic if its restriction to each affine piece $X \cap\left(\mathbf{P}^{n}-H_{i}\right)$ is semialgebraic, for $0 \leq i \leq n$.

\section{Algebraic neighborhoods of affine algebraic sets.}

Definition 1.1. Let $M$ be an algebraic set in $\mathbf{R}^{n}$ and let $X$ be a compact algebraic subset of $M$ with $M-X$ nonsingular. An (algebraic) rug function for $X$ in $M$ is a proper polynomial function $\alpha: M \rightarrow \mathbf{R}$ such that $\alpha(x) \geq 0$ for $x \in M$ and $\alpha^{-1}(0)=X$.

Recall that a function is proper if the inverse image of every compact set is compact. To say that $\alpha$ is a polynomial function means simply that it is the restriction of a polynomial on $\mathbf{R}^{n}$.

LEMMA 1.2. Let $f: \mathbf{R}^{n} \rightarrow \mathbf{R}$ be a polynomial function with $f^{-1}(0)$ compact and $f(x) \geq 0$ for all $x$. Then there is a proper polynomial function $g: \mathbf{R}^{n} \rightarrow \mathbf{R}$ with $g^{-1}(0)=f^{-1}(0)$ and $g(x) \geq 0$ for all $x$.

Proof. We may assume, without loss of generality, that $f(0)=0$. Let $\alpha:[0, \infty) \rightarrow \mathbf{R}$ be the function defined by

$$
\alpha(r)=\min _{|x|=r}\{f(x)\}
$$

It is not hard to see that $\alpha$ is a semialgebraic function. If $\lim _{r \rightarrow \infty} \alpha(r)=0$, then there is a positive integer $p$ and a positive number $b$ such that $\alpha(r) \geq b r^{-p}$ for large $r$ : Let $\beta(s)=\alpha\left(s^{-1}\right)$. Near $s=0$ the function $\beta$ has a fractional power series expansion

$$
\beta(s)=a s^{p / q}+a_{1} s^{(p+1) / q}+\cdots
$$

for some $a>0$ and some positive integers $p$ and $q$. Choose $b$ such that $0<b<a$. For small $s, \beta(s) \geq b s^{p / q} \geq b s^{p}$. Thus for large $r, \alpha(r) \geq b r^{-p}$. Now let $g$ be the polynomial function $g(x)=|x|^{2 p} f(x)$. Then $g^{-1}(0)=f^{-1}(0)$, and $g$ is proper since

$$
|g(x)| \geq|x|^{2 p} \alpha(|x|) \geq b|x|^{p} .
$$


A function $f$ as in the lemma may not be proper, as was pointed out to me by $\mathbf{H}$. King. For example, let

$$
f(x, y)=\left(x^{2}+y^{2}\right)\left(\left(y\left(x^{2}+1\right)-1\right)^{2}+y^{2}\right) .
$$

Then $f^{-1}(0)=0$ but $f\left(x,\left(1+x^{2}\right)^{-1}\right) \rightarrow 0$ as $x \rightarrow \infty$.

Corollary 1.3. Any set $X$ as in Definition 1.1 has a rug function.

Proof. It suffices to take $M=\mathbf{R}^{n}$ since the restriction of a rug function is a rug function. If

$$
X=\left\{f_{1}=\cdots=f_{k}=0\right\}
$$

then $X=\{f=0\}$ where $f=f_{1}^{2}+\cdots+f_{k}^{2}$, and the function $g$ as constructed in the above lemma for $f$ is a rug function for $X$.

LEMMA 1.4. A rug function has a finite number of critical values.

Proof. See [Milnor 2, Corollary 2.8].

Definition 1.5. Let $M$ and $X$ be as in Definition 1.1. A subset $T$ with $X \subset T \subset M$ is an algebraic neighborhood of $X$ in $M$ if $T=\alpha^{-1}[0, \delta]$, for some rug function $\alpha$ and some positive number $\delta$ smaller than all nonzero critical values of $\alpha$.

Suppose, for example, that $X=\{p\}$ is an isolated singular point of $M$. The boundary of an algebraic neighborhood $T$ of $p$ in $M$ is called the link of the singularity of $M$ at $p$. One usually takes the rug function

$$
\alpha(x)=|x-p|^{2} .
$$

Proposition 1.6. Let $T$ be an algebraic neighborhood of $X$ in $M$. Then the inclusion $X \subset T$ is homotopy equivalence.

Proof. Since $X$ and $M$ can be triangulated, $X$ has a basis of closed neighborhoods $X \subset \cdots \subset U_{n} \subset \cdots \subset U_{1} \subset M$ with each $U_{i}$ homotopy equivalent to $X$. Since the function $\alpha$ is proper, the sets $T_{n}=\alpha^{-1}[0, \delta / n]$ for $n=1,2, \ldots$ also form a neighborhood basis of $X$. Choose $n_{1}$ and $n_{2}$ such that $X \subset T_{n_{2}} \subset U_{n_{1}} \subset T_{1}$. The inclusion $T_{n_{2}} \subset T_{1}$ is a homotopy equivalence, since we may push $T_{n_{2}}$ out to $T_{1}$ using a gradient vector field of $\alpha$. Thus the inclusion $T_{n_{2}} \subset U_{n_{1}}$ induces an isomorphism of homotopy groups and hence is a homotopy equivalence.

Let $\alpha: M \rightarrow \mathbf{R}$ be a rug function for $X \subset M \subset \mathbf{R}^{n}$. The usual Riemannian metric on $\mathbf{R}^{n}$ induces a metric on the manifold $M-X$, and this metric may be used to define the gradient vector field $\operatorname{grad} \alpha$ of $\alpha$ on $M-X$. In fact, $\operatorname{grad} \alpha$ is a semialgebraic function on $M-X$, since grad $\alpha(x)$ is the image of the projection of the vector $\left(\partial \alpha / \partial x_{1}, \ldots, \partial \alpha / \partial x_{n}\right)$ to the tangent plane of $M-X$ at $x$.

Proposition 1.7 (UniQueness of Algebraic neighborhoods). Let $T_{1}$ and $T_{2}$ be algebraic neighborhoods of $X$ in $M$. Then there is a continuous family of homeomorphisms $h_{t}: M \rightarrow M$ for $0 \leq t \leq 1$ such that

a. $h_{0}$ is the identity,

b. $h_{t} \mid X$ is the identity, for all $t$,

c. $h_{1}\left(T_{1}\right)=T_{2}$, and $h_{1}$ is a smooth diffeomorphism of $T_{1}-X$ onto $T_{2}-X$. 
Proof. Let $T_{i}=\alpha_{i}^{-1}\left[0, \delta_{i}\right]$ for some rug function $\alpha_{i}$, for $i=1,2$.

When $\alpha_{1}=\alpha_{2}$ (and assuming without loss of generality that $\delta_{1}<\delta_{2}$ ), we may use the gradient vector field of $\alpha_{1}$ to push $T_{1}$ out to $T_{2}$. This is a standard technique of Morse theory [Milnor 1, Theorem 3.1]. In the case $\alpha_{1} \neq \alpha_{2}$, we need the following lemma. (Compare [Milnor 2, Corollary 3.4].)

LEMMA 1.8. There is a neighborhood $U$ of $X$ in $M$ such that $\operatorname{grad} \alpha_{1}$ and $\operatorname{grad} \alpha_{2}$ are nonzero and do not point in opposite directions on $U-X$.

ProOF. Let

$$
\begin{aligned}
Y=\{x \in M-X: & \operatorname{grad} \alpha_{1}(x) \text { and } \operatorname{grad} \alpha_{2}(x) \text { are both } \\
& \text { nonzero and point in opposite directions }\} .
\end{aligned}
$$

The set $Y$ is semialgebraic, since it is the intersection of the set of $x \in M-X$ where the inner product $\left\langle\operatorname{grad} \alpha_{1}(x), \operatorname{grad} \alpha_{2}(x)\right\rangle$ is negative with the set of $x \in M-X$ where grad $\alpha_{1}(x)$ and grad $\alpha_{2}(x)$ are linearly dependent, and these sets are semialgebraic. It suffices to show that $X$ does not intersect the closure of $Y$. Suppose this is false. Then by the curve selection lemma [Milnor 2, 3.1] there is a real analytic curve $\beta:[0, \varepsilon) \rightarrow M$ with $\beta(0) \in X$ and $\beta(t) \in Y$ for $t>0$. Near $t=0, \alpha_{i} \beta(t)$ is an increasing function of $t$, so $d \alpha_{i} \beta(t) / d t$ is positive there. However, this derivative equals $\left\langle\operatorname{grad} \alpha_{i}\right.$, $d \beta / d t\rangle$, and $\operatorname{grad} \alpha_{1}$ is a negative multiple of $\operatorname{grad} \alpha_{2}$ on $Y$, a contradiction.

Choose $\delta_{2}^{\prime}>0$ such that $\alpha_{2}^{-1}\left[0, \delta_{2}^{\prime}\right] \subset U$ and then choose $\delta_{1}^{\prime}>0$ such that $\alpha_{1}^{-1}\left[0, \delta_{1}^{\prime}\right]$ is contained in the open set $\alpha_{2}^{-1}\left[0, \delta_{2}^{\prime}\right)$. By the first part of the proof, the sets $\alpha_{i}^{-1}\left[0, \delta_{i}\right]$ and $\alpha_{i}^{-1}\left[0, \delta_{i}^{\prime}\right]$ are isotopic, for $i=1,2$. Hence it suffices to find an isotopy of $\alpha_{1}^{-1}\left[0, \delta_{1}^{\prime}\right]$ to $\alpha_{2}^{-1}\left[0, \delta_{2}^{\prime}\right]$. Let $S$ be the subset of $M$ defined by

$$
S=\alpha_{2}^{-1}\left[0, \delta_{2}^{\prime}\right]-\alpha_{1}^{-1}\left[0, \delta_{1}^{\prime}\right)
$$

and let $f: S \rightarrow[0,1]$ be defined by

$$
f(x)=\frac{\alpha_{1}(x)-\delta_{1}^{\prime}}{\left(\alpha_{1}(x)-\delta_{1}^{\prime}\right)+\left(\delta_{2}^{\prime}-\alpha_{2}(x)\right)} .
$$

Note that $f^{-1}(0)=\alpha_{1}^{-1}\left(\delta_{1}^{\prime}\right)$ and $f^{-1}(1)=\alpha_{2}^{-1}\left(\delta_{2}^{\prime}\right)$, that the denominator of $f$ is never zero on $S$, and that $f$ is proper. The function $f$ has no critical values, since its gradient is

$$
\frac{\left(\alpha_{1}(x)-\delta_{1}^{\prime}\right) \operatorname{grad} \alpha_{2}+\left(\delta_{2}^{\prime}-\alpha_{2}(x)\right) \operatorname{grad} \alpha_{1}}{\left[\left(\alpha_{1}(x)-\delta_{1}^{\prime}\right)+\left(\delta_{2}^{\prime}-\alpha_{2}(x)\right)\right]^{2}}
$$

and $\operatorname{grad} \alpha_{2}$ and $\operatorname{grad} \alpha_{1}$ are nonzero and never point in opposite directions on $U-X$. Let $u$ be a vector field on $S$ which projects under the derivative of $f$ to the vector field $\partial / \partial t$ on $[0,1]$. Integrating $u$ gives the required isotopy $h_{t}$.

The uniqueness theorem shows, for instance, that the algebraic neighborhood of $X$ in $M$ is independent of the embedding of $M$ in $\mathbf{R}^{n}$. Let $h$ be a map of $\mathbf{R}^{n}$ to itself with an inverse, both of which are defined by polynomials. Then $\alpha \circ h$ is a rug function for $h^{-1}(X)$ in $h^{-1}(M)$, so $\alpha \circ h$ defines an algebraic neighborhood of $h^{-1}(X)$, and this neighborhood is homeomorphic to the neighborhood of $X$ defined by $\alpha$. 
There are two more general types of rug functions for which the above theory works equally well. The first possibility is to replace the polynomial $\alpha$ by a rational function. These will be important when generalizing to projective space (§2). The second is as follows:

Definition 1.1 (BIS). Let $M$ be an algebraic set in $\mathbf{R}^{n}$ and let $X$ be a compact algebraic subset with $M-X$ nonsingular. An (analytic) rug function for $X$ in $M$ is a proper real analytic function $\alpha$ (defined on some neighborhood of $M$ in $\mathbf{R}^{n}$ ) such that $\alpha \geq 0, \alpha^{-1}(0)=X$, and $\alpha$ has a finite number of critical values.

The theory goes through just as before, except in the proof of the uniqueness theorem where the analytic curve selection lemma must be used.

For example, let $X$ and $M$ be as usual, and suppose that both $X$ and $M$ are smooth. The function $\alpha: M \rightarrow \mathbf{R}$ defined by

$$
\alpha(x)=(\text { distance in } M \text { from } x \text { to } X)^{2}
$$

is an analytic rug function for $X$ in $M$. The set $\alpha^{-1}([0, \delta])$ for small $\delta$ is fibered by geodesics of length $\sqrt{\delta}$ which start perpendicular to $M$, and hence is a smooth tubular neighborhood of $X$ in $M$. The uniqueness theorem then gives the following result:

Corollary 1.9. Let $X$ and $M$ be as above, and suppose that both $X$ and $M$ are smooth. Then every algebraic neighborhood of $X$ in $M$ is a smooth tubular neighborhood of $X$ in $M$.

\section{Algebraic neighborhoods of projective algebraic sets.}

Definition 2.1. Let $M$ be an algebraic set in real or complex projective space $\mathbf{P}^{n}$ and let $X$ be an algebraic subset of $M$ with $M-X$ nonsingular. An (algebraic) rug function for $X$ in $M$ is a rational function $\alpha: M \rightarrow \mathbf{R} \cup\{\infty\}$ such that $\alpha(x) \geq 0$ for $x \in M$ and $\alpha^{-1}(0)=X$.

Any such set $X$ as above has a rug function: As before, it suffices to show this for $M=\mathbf{P}^{n}$. If

$$
X=\left\{F_{1}=\cdots=F_{n}=0\right\}
$$

where $F_{i}$ is a homogeneous polynomial of degree $d_{i}$, let

$$
\alpha=\left[\left|F_{1}\right|^{2} / r^{d_{1}}\right]+\cdots+\left[\left|F_{k}\right|^{2} / r^{d_{k}}\right]
$$

where $r\left(x_{0}, \ldots, x_{n}\right)=\left|x_{0}\right|^{2}+\cdots+\left|x_{n}\right|^{2}$.

Again a rug function $\alpha$ has only a finite number of critical values: The set of critical points of $\alpha$ is a semialgebraic set, which may be written as a finite union of smooth manifolds. Since $\alpha$ is constant when restricted to each of these smooth manifolds, $\alpha$ has only a finite number of critical values.

LEMMA 2.2. $\mathbf{R P}^{n}$ may be embedded as an algebraic set in $\mathbf{R}^{(n+1)^{2}}$ in such a way that every rational function on $\mathbf{R P}^{n}$ is the restriction of a rational function on $\mathbf{R}^{(n+1)^{2}}$. There is a similar embedding of $\mathbf{C P}^{n}$ in $\mathbf{R}^{2(n+1)^{2}}$. 
Proof. Let $x_{0}, \ldots, x_{n}$ be homogeneous coordinates for $\mathbf{R P}^{n}$ and let $y_{i j}$ for $0 \leq i$, $j \leq n$ be coordinates for $\mathbf{R}^{(n+1)^{2}}$. The embedding is defined by

$$
y_{i j}=x_{i} x_{j} /\left(x_{0}^{2}+\cdots+x_{n}^{2}\right) \text {. }
$$

It is easy to see that this has the required properties. The rational function $\alpha\left(x_{0}, \ldots, x_{n}\right)$ on $\mathbf{R P}^{n}$ corresponds to the rational function $\alpha\left(y_{0 j}, \ldots, y_{n j}\right)$ on $\mathbf{R}^{(n+1)^{2}}$, for any $j$. (This embedding followed by the projection $\mathbf{R}^{(n+1)^{2}} \rightarrow \mathbf{R P}^{(n+1)^{2}-1}$ is the Veronese embedding of algebraic geometry.)

Similarly, if $z_{0}, \ldots, z_{n}$ are homogeneous coordinates for $\mathbf{C P}^{n}$ and $w_{i j}, 0 \leq i, j \leq n$ are complex coordinates for $\mathbf{C}^{(n+1)^{2}} \simeq \mathbf{R}^{2(n+1)^{2}}$, we let

$$
w_{i j}=z_{i} \bar{z}_{j} /\left(z_{0} \bar{z}_{0}+\cdots+z_{n} \bar{z}_{n}\right) \text {. }
$$

The uniqueness theorem for algebraic neighborhoods (Proposition 1.7) holds exactly as stated before. It is proved by using the above lemma to reduce to the affine case. Corollary 1.9 holds as well.

Application 2.3. Let $M$ be a nonsingular algebraic set in $\mathbf{A}^{n}$ and let $D$ be a suitably large ball about the origin of $\mathbf{A}^{n}$. Let $\bar{M} \subset \mathbf{P}^{n}$ be the projective completion of $M$ and let $H=\bar{M}-M$. Then $\bar{M}-\bar{M} \cap D$ is an algebraic neighborhood of $H$ in $\bar{M}$.

Proof. Let $\mathbf{A}^{n}$ have coordinates $x_{1}, \ldots, x_{n}$ and let $\mathbf{P}^{n}$ have coordinates $x_{0}, \ldots, x_{n}$. The large ball in $\mathbf{A}^{n}$ is $r(x) \leq N$ for some number $N$, where $r(x)=\left|x_{1}\right|^{2}+\cdots+$ $\left|x_{n}\right|^{2}$. Let $\alpha: \mathbf{P}^{n}-(1,0, \ldots, 0) \rightarrow \mathbf{R}$ be the extension to $\mathbf{P}^{n}$ of the inverse of the function $r$; in projective coordinates $\alpha$ may be written

$$
\alpha\left(x_{0}, \ldots, x_{n}\right)=\left|x_{0}\right|^{2} /\left(\left|x_{1}\right|^{2}+\cdots+\left|x_{n}\right|^{2}\right) .
$$

Clearly $\alpha$ is a rug function for $H$ in $\bar{M}$.

\section{Semialgebraic neighborhoods of affine semialgebraic sets.}

Definition 3.1. Let $M$ be a semialgebraic set in $\mathbf{R}^{n}$ and let $X \subset M$ be a compact semialgebraic subset. $A$ (semialgebriac) rug function for $X$ in $M$ is a proper semialgebraic function $\alpha: M \rightarrow \mathbf{R}$ such that $\alpha(x) \geq 0$ for $x \in M$ and $\alpha^{-1}(0)=X$.

Here are some examples of semialgebriac rug functions:

1. $M=\mathbf{R}^{n}, X=\{0\}, \alpha(x)=x_{1}^{2}+\cdots+x_{n}^{2}$.

2. $M=\mathbf{R}^{n}, X=\{0\}, \alpha(x)=\max \left\{\left|x_{1}\right|, \ldots,\left|x_{n}\right|\right\}$ (a PL function).

3. $M=\mathbf{R}^{2}, X=\{0\}, \alpha(x)=x_{1}^{213}+x_{2}^{2}$ (a semialgebraic function which is not smooth).

4. $M$ is an algebraic set with $X=\{0\}$ a nonisolated singular point, and $\alpha(x)=$ $x_{1}^{2}+\cdots+x_{n}^{2}$.

\section{LEMMA 3.2. Semialgebraic rug functions exist.}

Proof. The set $X=\left\{x \in \mathbf{R}^{n}: f(x) \geq 0\right\}$ has rug function $\alpha(x)=\max (-f(x), 0)$. If the semialgebraic sets $X_{1}$ and $X_{2}$ have rug functions $\alpha_{1}$ and $\alpha_{2}$, then the set $X_{1} \cup X_{2}$ has rug function $\alpha_{1} \alpha_{2}$ (or $\min \left(\alpha_{1}, \alpha_{2}\right)$ ), and the set $X_{1} \cap X_{2}$ has rug function $\alpha_{1}+\alpha_{2}$ (or $\max \left(\alpha_{1}, \alpha_{2}\right)$ ). Thus every compact simplicial complex in $\mathbf{R}^{n}$ has a rug function. Now let $M$ and $X$ be as in Definition 3.1. By the triangulation theorem, there is a simplicial decomposition $\mathbf{R}^{n}=\bigcup_{i \in I} \Delta_{i}$ and a semialgebraic automorphism $\sigma$ of 
$\mathbf{R}^{n}$ such that $X=\bigcup_{i \in J} \sigma\left(\Delta_{i}\right)$ for some $J \subset I$. Since $X$ is closed, $K=\bigcup_{i \in J} \Delta_{i}$ is a simplicial complex in $\mathbf{R}^{n}$. Let $\alpha$ be a rug function for $K$ in $\mathbf{R}^{n}$. Then $\alpha \circ \sigma^{-1}$ is a rug function for $X$ in $\mathbf{R}^{n}$, and the restriction of this to $M$ is a rug function for $X$ in $M$.

Let $X$ be a subset of $\mathbf{R}^{n}$. A stratification of $X$ is a partition $X=\bigcup \Delta_{i}$ of $X$ into a finite collection of pairwise disjoint smooth submanifolds $\Delta_{i}$ of $\mathbf{R}^{n}$ satisfying the frontier condition: If $\bar{\Delta}_{i} \cap \Delta_{j} \neq \varnothing$, then $\bar{\Delta}_{i} \supset \Delta_{j}$. A Whitney stratification is a stratification satisfying Whitney's condition b [Mather, p. 2; Wall]. A substratification of a stratified set $X=\bigcup \Delta_{i}$ is a stratification $X=\bigcup \Delta_{j}^{\prime}$ such that each $\Delta_{j}^{\prime}$ is contained in some $\Delta_{i}^{\prime}$.

Two stratified sets $X=\bigcup \Delta_{i}$ and $Y=\bigcup \Gamma_{j}$ are isomorphic if there is a homeomorphism $f: X \rightarrow Y$ with the property that for each stratum $\Delta_{i}$ of $X$ the map $f \mid \Delta_{i}$ is a diffeomorphism of $\Delta_{i}$ to some stratum $\Gamma_{j}$ of $Y$.

Let $X$ be a semialgebraic set in $\mathbf{R}^{n}$. A semialgebraic stratification of $X$ is a stratification $X=\bigcup \Delta_{i}$ of $X$ with each $\Delta_{i}$ a nonsingular semialgebraic set. The dimension of $X$ is the maximum of the dimensions of the strata $\Delta_{i}$.

TheOREM (SEE E.G. [Wall]). Let $X_{1}, \ldots, X_{r}$ be semialgebraic sets in $\mathbf{R}^{n}$. Then there is a Whitney semialgebraic stratification of $\mathbf{R}^{n}$ such that each $X_{i}$ is a union of strata.

THEOREM 3.3 (STRATIFICATION OF MAPS). Let $X \subset \mathbf{R}^{n}$ be a semialgebraic set and let $f: X \rightarrow \mathbf{R}^{p}$ be a semialgebraic function. Then there is a Whitney semialgebraic stratification $X=\bigcup \Delta_{i}$ of $X$ such that $f \mid \Delta_{i}$ is real analytic and of constant rank, for each $i$.

We call this an $f$-stratification of $X$. This theorem is well known, but a proof is given here for lack of a reference. We need the following facts:

Fact 1. Let $X \subset \mathbf{R}^{n}$ be a semialgebraic set and let $f: X \rightarrow \mathbf{R}^{p}$ be a semialgebraic function. Then there is a Whitney semialgebraic stratification $X=\bigcup \Delta_{i}$ such that the graph of $f \mid \Delta_{i}$ is a nonsingular semialgebraic set, for each $i$.

Proof. Let $\Gamma \subset \mathbf{R}^{n} \times \mathbf{R}^{p}$ be the graph of $f$, and let $\Gamma=\bigcup \Gamma_{j}$ be a semialgebraic stratification of $\Gamma$. Let $\pi: X \times \mathbf{R}^{p} \rightarrow X$ be the projection to the first factor. By the Tarski-Seidenberg theorem, each set $\pi\left(\Gamma_{j}\right)$ is semialgebraic. Then let $X=\bigcup \Delta_{i}$ be a Whitney semialgebraic stratification of $X$ such that each $\pi\left(\Gamma_{j}\right)$ is a union of strata.

Fact 2. Let $X$ be a nonsingular semialgebraic set and let $f: X \rightarrow \mathbf{R}^{p}$ be a function whose graph is a nonsingular semialgebraic set. Then the set of points of $X$ where $f$ is not differentiable is contained in a closed semialgebraic set with dimension less than the dimension of $X$.

Proof. This set is contained in the set of critical values of the function which projects the graph of $f$ onto $X$.

Fact 3. Let $U$ be an open subset in $\mathbf{R}^{m}$ and let $f: U \rightarrow \mathbf{R}^{p}$ be a differentiable function whose graph $\Gamma$ is a real analytic submanifold of $\mathbf{R}^{m} \times \mathbf{R}^{p}$. Then $f$ is a real analytic function.

Proof. The projection of $\Gamma$ to $\mathbf{R}^{m}$ is a real analytic map whose derivative is invertible. Hence its inverse is real analytic. The $\operatorname{map} f$ is the composition of this inverse with the projection to $\mathbf{R}^{p}$. 
Fact 4. Let $U \subset \mathbf{R}^{n}$ be a nonsingular semialgebraic set of dimension $m$ and let $f: U \rightarrow \mathbf{R}^{p}$ be a differentiable function whose graph in $\mathbf{R}^{n} \times \mathbf{R}^{p}$ is semialgebraic. Then there is a Whitney semialgebraic stratification $U=\bigcup U_{i}$ such that $f \mid U_{i}$ has constant rank, for each $i$.

Proof. The derivative $D f: U \rightarrow T U$ of $f$ is a semialgebraic function. There is a Whitney semialgebraic stratification of $m \times p$ real matrices such that each stratum consists of matrices of constant rank. Hence the tangent bundle $T U$ has such a stratification, and this is pulled back to $U$.

ProOF OF THE THEOREM. The proof is by induction on the dimension of $X$. By Fact 1 , we may assume that $X$ and the graph of $f$ are nonsingular semialgebraic sets. By Fact 2, there is a closed semialgebraic set $Y$ in $X$ with $\operatorname{dim} Y<\operatorname{dim} X$ such that $f$ is differentiable on $X-Y$. The set $Y$ has an $f$-stratification by inductive hypothesis. By Fact 3, $f$ is real analytic on $X-Y$, and by Fact 4 there is a stratification of $X-Y$ such that $f$ has constant rank on each stratum. Combining these two stratifications gives an $f$-stratification of $X$.

Definition 3.4. Let $M$ be a semialgebraic set in $\mathbf{R}^{n}$ and let $X \subset M$ be a compact semialgebraic set. A set $T$ with $X \subset T \subset M$ is a semialgebraic neighborhood of $X$ in $M$ if there is a semialgebraic rug function $\alpha$ and a suitably small positive number $\delta$ such that $T=\alpha^{-1}[0, \delta]$.

The problem is to determine how small $\delta$ should be. Let $M=\bigcup \Delta_{i}$ be an $\alpha$ stratification of $M$ such that $X$ is a union of strata. Let

$$
J=\left\{i: \Delta_{i} \cap X=\varnothing \text { and } \bar{\Delta}_{i} \cap X \neq \varnothing\right\} .
$$

Then $\alpha$ has rank one on each $\Delta_{i}$ for $i \in J$ : If $\alpha \mid \Delta_{i}$ had rank 0 then $\alpha \mid \Delta_{i}$ would be constant and in fact would have value 0 , which contradicts $\Delta_{i} \cap X=\varnothing$. Now choose $\delta$ small enough so that $0<\alpha(x) \leq \delta$ implies that $x \in \bigcup_{i \in J} \Delta_{i}$.

As for algebraic neighborhoods, the inclusion $X \subset T$ is a homotopy equivalence. Two semialgebraic sets $X$ and $Y$ are $S$-isomorphic if there are Whitney semialgebraic stratifications of $X$ and $Y$ which are isomorphic as stratifications.

Proposition 3.5 (UniQueness of Semialgebraic neighborhoods). Let $T_{1}$ and $T_{2}$ be semialgebraic neighborhoods of $X$ in $M$. Then there is a continuous family of homeomorphisms $h_{t}: M \rightarrow M$ for $0 \leq t \leq 1$ such that

a. $h_{0}$ is the identity,

b. $h_{t} \mid X$ is the identity, for all $t$,

c. $h_{1}\left(T_{1}\right)=T_{2}$, and $h_{1}$ is an $S$-isomorphism of $T_{1}$ and $T_{2}$.

Proof. For $j=1,2$, let $T_{j}=\alpha_{j}^{-1}\left[0, \delta_{j}\right]$ for some semialgebraic rug function $\alpha_{j}$ and $\alpha_{j}$-stratification $M=\bigcup \Delta_{i}^{j}$ as above. Let $M=\bigcup \Delta_{i}$ be a Whitney semialgebraic stratification of $M$ which is a substratification of $\bigcup \Delta_{i}^{1}$ and $U \Delta_{i}^{2}$.

First suppose that $\alpha_{1}=\alpha_{2}$ and that $\delta_{1}<\delta_{2}$. Let $S=\alpha_{1}^{-1}\left(0, \delta_{2}^{\prime}\right)$ for some $\delta_{2}^{\prime}>\delta_{2}$ with the property that $\alpha_{1}^{-1}\left[0, \delta_{2}^{\prime}\right]$ is still a semialgebraic neighborhood. The Whitney semialgebraic stratification of $M$ restricts to $S$. Consider the function $\alpha_{1}: S \rightarrow R$. The gradient vector field of $\alpha_{1} \mid \Delta_{i}$ is nonzero on the set $S \cap \Delta_{i}$, for each $i \in J$. Thom's first isotopy lemma then says that there is a controlled vector field generating a one- 
parameter group of maps which gives an isomorphism of the stratified sets $\alpha_{1}^{-1}\left[0, \delta_{1}\right]$ and $\alpha_{1}^{-1}\left[0, \delta_{2}\right]$ [Mather].

Now we return to the general case $\alpha_{1} \neq \alpha_{2}$. The following lemma is proved exactly as Lemma 1.8 is proved.

Lemma 3.6. Let $\Delta \subset \mathbf{R}^{n}$ be a nonsingular semialgebraic set and let $p \in \mathbf{R}^{n}$ be in the closure of $\Delta$. Let $\alpha_{1}, \alpha_{2}: \Delta \rightarrow \mathbf{R}$ be differentiable functions with semialgebraic graphs which vanish at $p$. Then there is a neighborhood of $p$ in $\bar{\Delta}$ on which $\operatorname{grad} \alpha_{1}$ and $\operatorname{grad} \alpha_{2}$ do not point in opposite directions unless one of them vanishes.

By applying Lemma 3.6 to all $\Delta_{i}$ for $i \in J$, we find a neighborhood $U$ of $X$ such that $\operatorname{grad}\left(\alpha_{1} \mid \Delta_{i}\right)$ and $\operatorname{grad}\left(\alpha_{2} \mid \Delta_{i}\right)$ are nonzero and do not point in opposite directions on $(U-X) \cap \Delta_{i}$, for all $i \in J$. Choose $\delta_{2}^{\prime}>0$ such that $\alpha_{2}^{-1}\left[0, \delta_{2}^{\prime}\right] \subset U$ and choose $\delta_{1}^{\prime}>0$ such that $\alpha_{1}^{-1}\left[0, \delta_{1}^{\prime}\right] \subset \alpha_{2}^{-1}\left[0, \delta_{2}^{\prime}\right)$. By the first part of the proof, the stratified sets $\alpha_{j}^{-1}\left[0, \delta_{j}\right]$ and $\alpha_{j}^{-1}\left[0, \delta_{j}^{\prime}\right]$ with stratifications $\bigcup_{i} \Delta_{i}^{j}$ are isomorphic, for $j=1,2$. Also, the stratified sets $\alpha_{j}^{-1}\left[0, \delta_{j}^{\prime}\right]$ with stratifications $\bigcup_{i} \Delta_{i}^{j}$ and $\bigcup_{i} \Delta_{i}$ are $S$-isomorphic, for $j=1,2$. We will show that the stratified sets $\alpha_{1}^{-1}\left[0, \delta_{1}^{\prime}\right]$ and $\alpha_{2}^{-1}\left[0, \delta_{2}^{\prime}\right]$ with stratifications $U \Delta_{i}$ are isomorphic. Define $S$ and $f: S \rightarrow[0,1]$ as in the proof of Proposition 1.7. The set $S$ is a stratified subset of $\mathbf{R}^{n}$ and the map $f$ is proper and has no critical values when restricted to each stratum of $S$. As in the first part of the proof, we can find a controlled vector field on $S$ which generates a one-parameter group of maps taking $f^{-1}(0)=\alpha_{1}^{-1}\left(\delta_{1}^{\prime}\right)$ to $f^{-1}(1)=\alpha_{2}^{-1}\left(\delta_{2}^{\prime}\right)$.

Proposition 3.7. Let $X \subset T \subset M$ be finite simplicial complexes in $\mathbf{R}^{n}$, and suppose that $T$ is a PL regular neighborhood of $X$ in $M$. Then $T$ is a semialgebraic neighborhood of $X$ in $M$.

Proof. One way of constructing a PL regular neighborhood of $X$ in $M$ is the following [Hudson, p. 51]: Subdivide $X$ so that it is a full subcomplex of $M$. Let $\alpha$ : $M \rightarrow \mathbf{R}$ be a linear function with $\alpha \geq 0$ and $\alpha^{-1}(0)=X$. Choose $\delta>0$ such that $\delta<\alpha(v)$, for any vertex $v$ of $M-X$. Then $\alpha^{-1}[0, \delta]$ is a PL regular neighborhood of $X$ in $M$. This is a semialgebraic neighborhood as well. By PL uniqueness [Hudson, p. 57], every PL neighborhood can be constructed this way.

It would be interesting to know whether the following is true or not: Let $X \subset T \subset$ $M \subset \mathbf{R}^{n}$ be closed semialgebraic sets with $X$ compact and $T$ a semialgebraic neighborhood of $X$ in $M$. Let $\alpha$ be a semialgebraic automorphism of $\mathbf{R}^{n}$ such that $\sigma(X) \subset$ $\sigma(T) \subset \sigma(M)$ are simplicial complexes in $\mathbf{R}^{n}$. Then $\sigma(T)$ is a PL regular neighborhood of $\sigma(X)$ in $\sigma(M)$. This would be true, for instance, if $S$-isomorphic simplicial complexes were PL-isomorphic.

Application 3.8 (polydisk links of singularities). Let $M$ be a real or complex algebraic set in $\mathbf{R}^{n}$ or $\mathbf{C}^{n}$ (with coordinates $x=\left(x_{1}, \ldots, x_{n}\right)$ ) which contains the origin 0 . The (semialgebraic) link of 0 in $M$ is $\partial T$, where $T$ is any semialgebraic neighborhood of 0 in $M$. For example, we could use the rug functions

$$
\alpha_{1}(x)=\left|x_{1}\right|^{2}+\cdots+\left|x_{n}\right|^{2}
$$

or

$$
\alpha_{2}(x)=\max \left(\left|x_{1}\right| / \varepsilon_{1}, \ldots,\left|x_{n}\right| / \varepsilon_{n}\right)
$$


where $\varepsilon_{1}, \ldots, \varepsilon_{n}$ are fixed positive numbers. By the uniqueness theorem these links are $S$-isomorphic. For example, let $M$ be a complex algebraic curve with one branch at 0 , and suppose that the algebraic tangent space at 0 to this branch is the $x_{1}$-axis. Let $\varepsilon_{1}=\varepsilon_{2}=1$ and let $T=\alpha_{2}^{-1}[0, \delta]$. For small $\delta, M \cap \partial T$ lies in the part of $\partial T$ defined by $\left|x_{1}\right|=\delta, x_{2} \leq \delta$. An obvious generalization of the uniqueness theorem to pairs shows that the pair $(\partial T, \partial T \cap M)$ is $S$-isomorphic to $\left(S_{\delta}^{3}, S_{\delta}^{3} \cap M\right)$, where $S_{\delta}^{3}$ is the 3 -sphere of radius $\delta$ about 0 . The polydisk neighborhood is easier for computations [Kähler].

Next we generalize Milnor's fibration theorem [Milnor 2, §4] from polynomials on $\mathbf{C}^{n}$ to polynomials on an arbitrary complex variety.

Let $M$ be a complex set in $\mathbf{C}^{n}$, let $p$ be a point of $M$ and let $f: M \rightarrow \mathbf{C}$ be a complex polynomial function with $f(p)=0$. Let $N=f^{-1}(0)$. Let $D_{\delta}^{2 n}$ be the closed disk of radius $\delta$ about $p$ in $\mathbf{C}^{n}$ and let $S_{\delta}^{2 n-1}$ be its boundary.

TheOREM 3.9 (Fibration THEOREM). For suitably small positive $\delta$ and $\eta$, the space

$$
E=(M-N) \cap S_{\delta}^{2 n-1}
$$

is the total space of a fiber bundle over the circle whose fiber $F$ is homeomorphic to $f^{-1}(\eta) \cap D_{\delta}^{2 n}$.

Proof. The function $\alpha(x)=|x-p|$ is a rug function for $p$ in the pair $(M, N)$. (By this we mean a rug function for $p$ in $M$ and $p$ in $N$.) Choose $\delta$ small enough so that $(M, N) \cap D_{\delta}^{2 n}$ is a semialgebraic neighborhood of $p$ in $(M, N)$. Next, the function $|f|$ is a semialgebraic rug function for $N \cap\left(D_{\delta}^{2 n}, S_{\delta}^{2 n-1}\right)$ in $M \cap\left(D_{\delta}^{2 n}, S_{\delta}^{2 n-1}\right)$. Choose $\eta$ small enough so that the function $f$ restricted to any stratum of $M \cap\left(D_{\delta}^{2 n}, S_{\delta}^{2 n-1}\right)$ has no critical values in $D_{\eta}^{2}-0$. Then $f^{-1}\left(D_{\eta}^{2}\right) \cap\left(D_{\delta}^{2 n}, S_{\delta}^{2 n-1}\right)$ is a semialgebraic neighborhood of $N \cap\left(D_{\delta}^{2 n}, S_{\delta}^{2 n-1}\right)$ in $M \cap\left(D_{\delta}^{2 n}, S_{\delta}^{2 n-1}\right)$. By Thom's first isotopy lemma [Mather], the map of pairs

$$
f: f^{-1}\left(D_{\eta}^{2}-0\right) \cap\left(D_{\delta}^{2 n}, S_{\delta}^{2 n-1}\right) \rightarrow\left(D_{\eta}^{2}-0\right)
$$

is a locally trivial fiber bundle with fiber $f^{-1}(\eta) \cap\left(D_{\delta}^{2 n}, S_{\delta}^{2 n-1}\right)$. Hence

$$
E^{\prime}=\left(f^{-1}\left(D_{\eta}^{2}-0\right) \cap S_{\delta}^{2 n-1}\right) \cup\left(f_{\eta}^{-1}\left(S^{1}\right) \cap D_{\delta}^{2 n}\right)
$$

is a fiber bundle over $S_{\eta}^{1}$ with fiber homeomorphic to $f^{-1}(\eta) \cap D_{\delta}^{2 n}$.

Let $\beta: M \rightarrow \mathbf{R}$ be defined by

$$
\beta(x)=\max \{\eta|x-p|, \delta|f(x)|\} .
$$

Note that $\beta^{-1}([0, \eta \delta])$ is the closed set bounded by $E^{\prime}$. The function $\beta$ is also a rug function for $p$ in $(M, N)$. By making $\delta$ and $\eta$ smaller if necessary, we may assume that $\beta^{-1}([0, \eta \delta])$ is a semialgebraic neighborhood of $p$ in $(M, N)$. By the uniqueness theorem, the sets $\alpha^{-1}(\delta)$ and $\beta^{-1}(\eta \delta)$ are homeomorphic, and (looking at the proof), this homeomorphism fixes their common intersection $f^{-1}\left(D_{\eta}^{2}\right) \cap S_{\delta}^{2 n-1}$. Thus $E$ and $E^{\prime}$ are homeomorphic, so $E$ is also a fiber bundle over $S_{\eta}^{1}$ with fiber homeomorphic to $f^{-1}(\eta) \cap$ $D_{\delta}^{2 n}$. 
(A result similar to Theorem 3.9 has also been Obtained by Lê Dũng Tráng.)

The fibration theorem can now be used to relate the connectivity of the fiber $F$ with the connectivity of the link of $p$ in $M$ :

Corollary 3.10 (CONNECTIVITY OF THE FIBER). Suppose in addition that $M$ has dimension $m$ and is a subset of $C^{m+r}(i . e ., n=m+r)$, and that $p$ is an isolated singularity. Then $F$ is $(m-r-1)$-connected.

Proof. Let $K=M \cap S^{2 n-1}$. According to a local connectivity theorem [Hamm], $K$ is $(m-r-1)$-connected. Furthermore, the complex $(m-1)$-dimensional fiber $F$ is diffeomorphic to $f^{-1}(\eta) \cap D_{\delta}^{2 n}$, which by the usual Morse theory argument is obtained from a $2(m-1)$-disk by attaching handles of index $\leq m-1$. Also, $K-F$ is homotopy equivalent to $F$. The argument is now similar to [Milnor, §6]: Consider the exact sequence

$$
\rightarrow H_{i+1}(K, F) \rightarrow \tilde{H}_{i}(F) \rightarrow \tilde{H}_{i}(K) \rightarrow H_{i}(K, F) \rightarrow .
$$

By duality,

$$
H_{i+1}(K, F) \simeq H^{2 m-2-i}(K-F) \simeq H^{2 m-2-i}(F)=0
$$

for $i<m-1$. Also, $\tilde{H}_{i}(K)=0$ for $i<m-r$. Thus $\tilde{H}_{i}(F)=0$ for $i<m-r$; an argument as in [Milnor, p. 57] shows that $F$ is simply-connected as well.

\section{Semialgebraic neighborhoods of projective algebraic sets.}

Definition 4.1. Let $M$ be an algebraic set in $\mathbf{P}^{n}$ and let $X$ be an algebraic subset. A semialgebraic rug function for $X$ in $M$ is a semialgebraic function $\alpha: M \rightarrow \mathbf{R}$ with $\alpha(x) \geq 0$ for $x \in M$ and $\alpha^{-1}(0)=X$.

Semialgebraic regular neighborhoods are defined just as in the affine case, and a uniqueness theorem similar to 3.5 is proved in the same way.

This theory can obviously be extended to (appropriately defined) projective semialgebraic sets, but we do not need this.

Application 4.2. Let $M$ be a real or complex algebraic set in $\mathbf{P}^{n}$ with singular locus $S \subset M$, and let $T^{\prime}$ be an algebraic neighborhood of $S$ in $M$. Let $\pi: N \rightarrow M$ be a resolution of singularities. ( $N$ is a nonsingular algebraic set, $\pi$ is a proper algebraic map, $\pi \mid\left(N-\pi^{-1}(S)\right)$ is an algebraic isomorphism, and $N-\pi^{-1}(S)$ is dense in $N$.) Assume further that $\pi^{-1}(S)$ is a union $D_{1} \cup \cdots \cup D_{r}$ of nonsingular codimension-one algebraic sets $D_{i}$ with transverse intersections. Let $T_{i}$ be a suitably small tubular neighborhood of $D_{i}$. Then the stratified sets $\partial T^{\prime}$ and $\partial\left(\bigcup T_{i}\right)$ are $S$-isomorphic, and hence in particular homeomorphic. In fact, $\partial T^{\prime}$ is diffeomorphic to $\partial\left(U T_{i}\right)$ with its corners rounded (cf. [Mumford] for the case $\operatorname{dim} M=2$ ).

Proof. We may assume without loss of generality that each $T_{i}$ is an algebraic neighborhood of $D_{i}$. Then $\bigcup T_{i}$ is a semialgebraic regular neighborhood of $\bigcup D_{i}$. (If $T_{i}$ is defined by a rug function $\alpha_{i}$, then the rug function $\min \left(\alpha_{1}, \ldots, \alpha_{r}\right)$ gives $\bigcup T_{i}$.) Also $\pi^{-1}\left(T^{\prime}\right)$ is a regular neighborhood of $\bigcup D_{i}$ in $N$. By the uniqueness theorem, $\bigcup T_{i}$ and $\pi^{-1}\left(T^{\prime}\right)$ are isomorphic as stratified sets. Thus $\partial\left(\bigcup T_{i}\right)$ and $\partial\left(\pi^{-1}\left(T^{\prime}\right)\right)$, which is diffeomorphic to $\partial T^{\prime}$, are $S$-isomorphic. Furthermore, $\partial\left(\bigcup T_{i}\right)$ with its corners 
rounded is just $\left(\alpha_{1} \cdots \alpha_{r}\right)^{-1}(\delta)$, for small $\delta>0$. By the uniqueness theorem for algebraic neighborhoods, $\left(\alpha_{1} \cdots \alpha_{r}\right)^{-1}(\delta)$ is diffeomorphic to $\partial T^{\prime}$.

A similar argument shows the following result.

Application 4.3. Let $M$ be a complex manifold, let

$$
D_{\delta}=\{z \in \mathbf{C}:|z|<\delta\}
$$

and let $f: M \rightarrow D_{\delta}$ be a proper analytic map with the origin as its only possible critical value. Suppose further that $f^{-1}(0)$ is a union $D_{1} \cup \cdots \cup D_{r}$ of nonsingular codimension one submanifolds with transverse intersections. Let $T_{i}$ be a suitably small neighborhood of $D_{i}$ and let $T=T_{1} \cup \cdots \cup T_{r}$. Then $f^{-1}\left(D_{\eta}\right)$ for any $0<\eta<\delta$ is diffeomorphic to $T$ with its corners rounded.

5. A $\mathscr{C}^{\infty}$ counterexample. We will find smooth algebraic manifolds $K \subset M$ and $\mathscr{C}^{\infty}$ functions $\alpha_{1}, \alpha_{2}: M \rightarrow \mathbf{R}$ with 0 as their only critical value and such that $\alpha_{i} \geq 0$ and $\alpha_{i}^{-1}(0)=K$. However, $\alpha_{1}^{-1}(\delta)$ will not be diffeomorphic to $\alpha_{2}^{-1}(\delta)$ for $\delta \neq 0$.

Let $K$ and $K^{\prime}$ be algebraic manifolds which are $h$-cobordant but not diffeomorphic. Let $M=K \times \mathbf{R}$ and let $\alpha_{1}: M \rightarrow \mathbf{R}$ be the projection. Then $\alpha_{1}^{-1}(\delta)$ is diffeomorphic to $K$, for all $\delta$.

The map $\alpha_{2}$ is defined as follows: By the usual trick there is a diffeomorphism of $K \times \mathbf{R}$ to $K^{\prime} \times \mathbf{R}$ :

$\mathrm{K} \times \mathrm{I}$

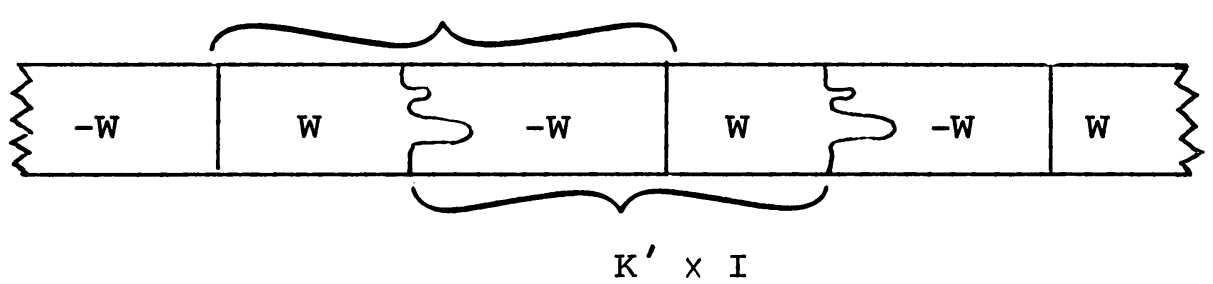

where $W$ is the cobordism of $K$ to $K^{\prime}$. This gives a diffeomorphism of $K \times(0, \infty)$ to $K^{\prime} \times(0, \infty)$. Extend this by reflection to a diffeomorphism of $K \times(\mathbf{R}-0)$ to $K^{\prime} \times$ $(\mathbf{R}-0)$, and let $\beta: K \times(\mathbf{R}-0) \rightarrow \mathbf{R}$ be this map followed by the projection. Extend $\beta$ to $K \times \mathbf{R}$ by mapping $K \times 0$ to 0 , and let $\alpha_{2}$ be a smoothing of $\beta$ [Church, pp. 95-96]. Then $\alpha_{2}^{-1}(\delta)$ is diffeomorphic to $K^{\prime}$ whenever $\delta \neq 0$.

I thank Henry King for helpful comments.

\section{BIBLIOGRAPHY}

W. Barth, Lokale Cohomologie bei isolierten Singularitäten analytischer Mengen, Schr. Math. Inst. Univ. Munster 2 (5) (1971).

P. Church, Differentiable open maps on n-manifolds, Trans. Amer. Math. Soc. 109 (1963), 87-100.

H. Hamm, On the vanishing of local homotopy groups for isolated singularites of complex spaces,

J. Reine Angew. Math. 323 (1981), 172-176.

H. Hironaka, Triangulations of algebraic sets, Proc. Sympos. Pure Math., vol. 29, Amer. Math.

Soc. Providence, R.I., 1975, pp. 165-185.

J. Hudson, Piecewise linear topology, Benjamin, New York, 1969. 
K. Kähler, Über die Verzweigung einer algebraischen Funktion zweier Veränderlichen in der Umgebung einen singularen Stelle, Math. Z. 30 (1929), 188-204.

J. Mather, Notes on topological stability, Harvard Univ. 1970.

J. Milnor, 1. Morse theory, Ann. of Math. Studies, no. 51, Princeton Univ. Press, Princeton, N.J., 1963.

2. Singular points of complex hypersurfaces, Ann. of Math. Studies, no. 61, Princeton Univ. Press, Princeton, N.J., 1968.

D. Mumford, The topology of normal singularities of an algebraic surface and a criterion for simplicity, Publ. Math. Inst. Hautes Etudes Scientifiques, no. 9 (1961).

R. Thom, Les structures différentiables des boules et des spheres, Colloque de Géométrie Différentielle Globale, Bruxelles 1958, Gauthier-Villars, Paris, 1959.

C. T. C. Wall, Regular stratifications, Dynamical Systems, Warwick 1974, Lecture Notes in Math., vol. 468, Springer-Verlag, Berlin and New York, 1975, pp. 332-344.

Department of Mathematics, Smtth College, Northampton, Massachusetts 01063 